\title{
Class III Polyhydroxybutyrate Synthase: Involvement in Chain Termination and Reinitiation $^{\dagger}$
}

\author{
Jiamin Tian, ${ }^{\ddagger}$ Anthony J. Sinskey, ${ }^{\S}$ and JoAnne Stubbe $*, \$, \S$ \\ Departments of Chemistry and Biology, Massachusetts Institute of Technology, 77 Massachusetts Avenue, \\ Cambridge, Massachusetts 02139
}

Received February 22, 2005; Revised Manuscript Received April 5, 2005

\begin{abstract}
Polyhydroxybutyrate (PHB) synthase catalyzes the polymerization of $(R)$-3-hydroxybutyryl$\mathrm{CoA}(\mathrm{CoA}=$ coenzyme A) into high molecular weight PHB. Recombinant wild-type $(\mathrm{wt})$ class III synthase from Allochromatium vinosum ( $\mathrm{PhaCPhaE}_{\mathrm{Av}}$ ), antibodies to this synthase and to $\mathrm{PHB}$, and $\left[{ }^{14} \mathrm{C}\right]-$ hydroxybutyryl-CoA (HB-CoA) have been used to detect oligomeric hydroxybutyrate (HB) units covalently bound to the synthase using sodium dodecyl sulfate-polyacrylamide gel electrophoresis analysis. Although a distribution of products is typically observed, short $(\mathrm{HB})_{n}$-bound synthases (designated species I) are most prevalent at low substrate to enzyme (S/E) ratios. Species I is similar to (HB) $)_{n}-\mathrm{PhaC}_{\mathrm{Av}}(n=3-10$ at minimum) recently identified using D302A-PhaCPhaE $\mathrm{Av}_{\mathrm{Av}}$ (Tian, J., Sinskey, A. J., and Stubbe, J. (2005) Biochemistry 44, 1495-1503). Species I is shown to be an intermediate in the elongation process of PHB synthesis in vitro. The reaction catalyzed by the wt synthase in vitro was further studied under two sets of conditions: at high (70000) and low (<200) S/E ratios. At high S/E ratios, kinetic analysis of the reaction of $\mathrm{HB}-\mathrm{CoA}$ with the wt synthase monitored using antibodies to $\mathrm{PhaCPhaE}_{\mathrm{Av}}$ and Western blotting revealed the disappearance of $\mathrm{PhaC}_{\mathrm{Av}}$ at early time points and its reappearance as the molecular weight of the PHB approached 1.8 MDa. At low S/E ratios, species I was observed to increase with time after complete consumption of all of the HB-CoA. The results from studies under both sets of conditions suggest that an inherent property of the synthase is chain termination and reinitiation.
\end{abstract}

Polyhydroxyalkanoate $\left(\mathrm{PHA}^{1}\right)$ synthases catalyze the polymerization of $(R)$-3-hydroxyalkanoate-CoA into high molecular weight polyoxoesters under nutrient-limited conditions in the presence of a carbon source $(1-4)$. PHA synthases are representative of enzymes involved in nontemplate-driven polymerization processes in which a watersoluble substrate is transformed into a water-insoluble granule or inclusion without a genetically determined template. The thermoplastic or elastomeric properties of PHAs (depending on the substrate) have provided the impetus for many research groups to study the mechanism and the regulation of the polymerization process, as well as the substrate specificity of the synthase. The long-range goal is to make materials with novel properties in an economically competitive fashion with oil-based polymers (5).

$\dagger$ This work was supported by NIH Grant GM49171 to J.S. and A.J.S. J.T. was supported by NIH Training Grant 5T32GM08334.

* To whom correspondence should be addressed. Phone: (617) 2531814. Fax: (617) 258-7247. E-mail: stubbe@ mit.edu.

Department of Chemistry.

$\S$ Department of Biology.

${ }^{1}$ Abbreviations: Abs, antibodies; CoA, coenzyme A; ESI-MS, electrospray ionization mass spectrometry; $\mathrm{HB}$, hydroxybutyrate; HBCoA, $(R)$-3-hydroxybutyryl-CoA; PHA, polyhydroxyalkanoate; $\mathrm{PhaC}_{\mathrm{We}}$, class I synthase from Wautersia eutropha; PhaCPhaE $\mathrm{Av}_{\mathrm{Av}}$, class III synthase from Allochromatium vinosum, $\mathrm{PhaC}$ and $\mathrm{PhaE}$ coexpressed and copurified; PHB, polyhydroxybutyrate; SA, specific activity; SDSPAGE, sodium dodecyl sulfate-polyacrylamide gel electrophoresis; $\mathrm{S} / \mathrm{E}$, substrate-to-enzyme ratio; sT, a trimer of 3-hydroxybutyrate in which the terminal hydroxyl is replaced with a hydrogen; sT-CoA, saturated trimer-CoA; wt, wild type.
The classification of PHA synthases, based on substrate specificity and subunit composition, has been described in detail in several reviews $(6,7)$. Our laboratory has focused on the class I and class III synthases that use $(R)-3$ hydroxybutyryl-CoA (HB-CoA) to form polyhydroxybutyrate (PHB). The class I synthase from the prototypical Wautersia eutropha $\left(\mathrm{PhaC}_{\mathrm{We}}\right)$ has a subunit molecular weight of $64 \mathrm{kDa}$ (8). The class III synthase from the prototypical Allochromatium vinosum is composed of two subunits, designated $\mathrm{PhaE}_{\mathrm{Av}}(\mathrm{MW} \approx 40 \mathrm{kDa})$ and $\mathrm{PhaC}_{\mathrm{Av}}(\mathrm{MW} \approx 39 \mathrm{kDa})(9)$. $\mathrm{PhaC}_{\mathrm{Av}}$ contains the active site involved in catalysis, while the role of $\mathrm{PhaE}_{\mathrm{Av}}$ remains unknown.

Three different mechanisms of initiation have been proposed for non-template-driven polymerization systems. The first involves priming by a protein other than the synthase itself and is exemplified by glycogenin in mammalian glycogen biosynthesis (10). The second involves priming with oligomers of the substrate unit and is exemplified by maltooligosaccharides ((glucose $)_{n}, n=2-7$ residues) in starch biosynthesis (11). The third involves self-priming by polymerase, a process exemplified by the bacterial glycogen synthase from Agrobacterium tumefaciens (12).

The mechanism of initiation by both the W. eutropha and A. vinosum synthases has been studied in some detail. Our initial examination of initiation used oligomers of HB-CoA. An analogue of $(\mathrm{HB})_{3}-\mathrm{CoA}$ in which the hydroxyl group of the terminal hydroxybutyrate (HB) unit was replaced by a hydrogen or tritium (sT-CoA, 1) was shown to function as a primer with both the class I and class III synthases (13, 


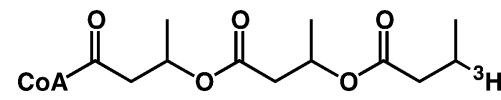

1

14). The studies revealed the importance of covalent catalysis using an active site cysteine. Furthermore, studies of the class III synthase with sT-CoA and small amounts of HB-CoA demonstrated that uniform loading of the synthase was possible with the sT-CoA and subsequently resulted in uniform elongation rates in the presence of $\mathrm{HB}-\mathrm{CoA}$ monomers. While these studies were mechanistically informative, the relationship of this mechanism of initiation to the one that occurs in vivo is unknown. Efforts to study the possibility of a self-priming initiation process with $\mathrm{HB}-\mathrm{CoA}$ which may be more likely in vivo, however, were unsuccessful because the rate of elongation was much greater than the rate of initiation in vitro, making the detection of intermediates difficult $(8,15)$. Most of the synthase remained unmodified, or if modified, the short (HB) ${ }_{n}$ linkages $(n=1$ or 2) were hydrolytically labile (14).

We recently studied a mutant class III synthase in which the aspartate, thought to be the general-base catalyst activating the 3'-hydroxyl group of HB-CoA for ester bond formation, was changed to an alanine. The reduced elongation rate (1/1000 of that of the wild type (wt)) allowed us for the first time to detect covalently bound $(\mathrm{HB})_{n}-\mathrm{PhaC}_{\mathrm{Av}}$ ( $n=3-10)$, using HB-CoA (15). These intermediates could be chased into PHB polymers. Results with this mutant synthase suggested that a self-priming mechanism is likely for initiation in vivo.

In this paper, we describe our continuing efforts to understand the initiation process catalyzed by the wt class I and class III synthases. We report the first direct evidence for $(\mathrm{HB})_{n}-\mathrm{PhaC}_{\mathrm{Av}}$ intermediates generated with the class III synthase and HB-CoA. These results provide support for the self-priming mechanism with the wt class III synthase. During these studies, we examined the interaction of this protein with its natural substrate at $\left[{ }^{14} \mathrm{C}\right] \mathrm{HB}-\mathrm{CoA} / \mathrm{enzyme}$ $(\mathrm{S} / \mathrm{E})$ ratios that varied from 5 to 70000 . The products generated from these experiments were analyzed by SDSPAGE with detection via autoradiography and Western blots using antibodies (Abs) to $\mathrm{PhaCPhaE}_{\mathrm{Av}}$ and $\mathrm{PHB}$. The results unexpectedly provided us with new insight into an additional role for this class III synthase. They support a model shown in Scheme 1 in which $\mathrm{PhaC}_{\mathrm{Av}}$ can sense the polymer chain length. In path $\mathrm{A}, \mathrm{PhaC}_{\mathrm{Av}}$ can catalyze transfer of most of the polymer chain to a second amino acid (designated as $\mathrm{Nu}$ in Scheme 1) concomitant with leaving a primed (HB) $n_{n^{-}}$ $\mathrm{PhaC}_{\mathrm{Av}}$ ready to reinitiate polymer formation. The polymer attached to the new site, surface exposed, is then rapidly hydrolyzed. In an alternative model, path $\mathrm{B}$, the $\mathrm{Nu}$ would act as a general-base catalyst to activate water for direct hydrolysis of the PHB chain, leaving a primed synthase. The possibility that a similar mechanism for termination/reinitiation occurs with the class I synthase is discussed.

\section{MATERIALS AND METHODS}

Materials. $(R, S)-\left[1-{ }^{14} \mathrm{C}\right] \mathrm{HB}-\mathrm{CoA}$ was obtained from American Radiolabeled Chemicals, Inc. $(R, S)-\left[1-{ }^{14} \mathrm{C}\right] \mathrm{HB}-\mathrm{CoA}$ was diluted with $(R)$-HB-CoA synthesized by the method of Yuan et al. (16). ( $S$ )-HB-CoA has previously been shown not to be a substrate for the synthase (16). Hecameg [6-O- $(\mathrm{N}-$ heptylcarbamoyl)methyl- $\alpha$-D-glucopyranoside] was obtained from Vegatec, Villejuif, France. Wt PhaCPhaE $\mathrm{Av}_{\mathrm{Av}}$ and C149A$\mathrm{PhaCPhaE}_{\mathrm{Av}}$ were overexpressed using plasmids pET-UM4 and pET-UM21, respectively, as previously described (14, 17). pKAS4 carrying $W$. eutropha $p h a C_{W e}$ has also been reported (8). Preparation of $\mathrm{Abs}$ to $\mathrm{PhaCPhaE}_{\mathrm{Av}}$ has been described previously (14). The Abs recognize both $\mathrm{PhaC}_{\mathrm{Av}}$ and $\mathrm{PhaE}_{\mathrm{Av}}$. Abs to PHB were a kind gift from Metabolix, Cambridge, MA; the antigen was prepared by covalently attaching a synthetic $\mathrm{PHB}$ oligomer, $(\mathrm{HB})_{8}$, to keyhole limpet hemocyanin. Edman sequencing of peptides and ESI-MS analysis of the peptides were carried out by the MIT Biopolymer Laboratory. HPLC was performed with a Rainin Dynamax model SD-200 HPLC system equipped with a Dynamax diode array detector (model PDA-1).

Purification of Recombinant PHA Synthases. Expression and purification of recombinant $\mathrm{PhaC}_{\mathrm{We}}$ was carried out as described previously (16). Recombinant wt and C149A$\mathrm{PhaCPhaE}_{\mathrm{Av}}$ were expressed and purified as described in Müh et al. (14). All synthases were assayed by the discontinuous method using 5,5'-dithiobis(2-nitrobenzoic acid) to monitor CoA release (14). $\mathrm{PhaC}_{\mathrm{We}}$ and $\mathrm{PhaCPhaE}_{\mathrm{Av}}$ have specific activities of 40 and 200 units/mg (unit $=\mu \mathrm{mol} /$ min), respectively. C149A-PhaCPhaE $\mathrm{Av}_{\mathrm{Av}}$ has no detectable activity.

Incubation of wt PhaCPhaE $E_{A v}$, C149A-PhaCPhaE $E_{A v}$, or $P h a C_{W e}$ with (R)-HB-CoA or $\left[{ }^{14} \mathrm{C}\right]-H B-C o A$. All reactions containing class III synthases were carried out in $20 \mathrm{mM}$ Tris/ $\mathrm{HCl}, \mathrm{pH} 7.8,50 \mathrm{mM} \mathrm{NaCl}$ with variable amounts of $\left[{ }^{14} \mathrm{C}\right] \mathrm{HB}-\mathrm{CoA}$ and were incubated at $30{ }^{\circ} \mathrm{C}$ for $5 \mathrm{~min}$, unless indicated otherwise. All reactions containing class I synthase were carried out in $150 \mathrm{mM} \mathrm{KP}, \mathrm{pH} 7.2,0.2 \%$ glycerol with variable amounts of substrate and were incubated at $25{ }^{\circ} \mathrm{C}$ for $1 \mathrm{~h}$. The details of each specific experiment, such as concentration of the synthase and substrate, and the specific activity (SA) of the substrate are described in the appropriate figure caption. Note that, throughout the text, the S/E ratio refers to the ratio of [substrate] to [monomeric PhaCPhaE $\mathrm{Av}_{\mathrm{Av}}$ $(\sim 80 \mathrm{kDa})]$ in the case of class III synthase or to [monomeric $\left.\mathrm{PhaC}_{\mathrm{We}}\right]$ in the case of class I synthase, unless indicated otherwise.

Analysis of Reaction Products by SDS-PAGE: Coomassie Staining, Autoradiography, and Western Blotting. All reactions were stopped by addition of an equal volume of Laemmli buffer (18) with no reducing reagent. Samples were not boiled. All samples were analyzed on $10 \%$ SDS-PAGE gels, followed by Coomassie staining, autoradiography, Western blotting, or a combination of these methods, as specified in the figure caption. The details of these methods have been described by Tian et al. (15). Abs raised against $\mathrm{PhaCPhaE}_{\mathrm{Av}}$ were used at a $1 / 1000$ dilution. Abs to PHB were also used at 1/1000 dilution.

Isolation by HPLC of Peptides from wt PhaCPhaE ${ }_{A v}$ Incubated with 1 equiv of $\left[{ }^{14} \mathrm{C}\right] \mathrm{HB}-\mathrm{CoA}$ Followed by Digestion with Trypsin. In a final volume of $112 \mu \mathrm{L}, 56 \mu \mathrm{M}$ PhaCPhaE $_{\mathrm{Av}}(500 \mu \mathrm{g})$ was reacted with $\left[{ }^{14} \mathrm{C}\right] \mathrm{HB}-\mathrm{CoA}(3.1$ $\times 10^{7} \mathrm{cpm} / \mu \mathrm{mol}$ ) at an $\mathrm{S} / \mathrm{E}$ ratio of $1 / 1$ in assay buffer for $5 \mathrm{~min}$ at room temperature. The reaction mixture was then processed following the procedure described previously (15) and analyzed by N-terminal sequencing and ESI-MS. The 
Scheme $1^{a}$

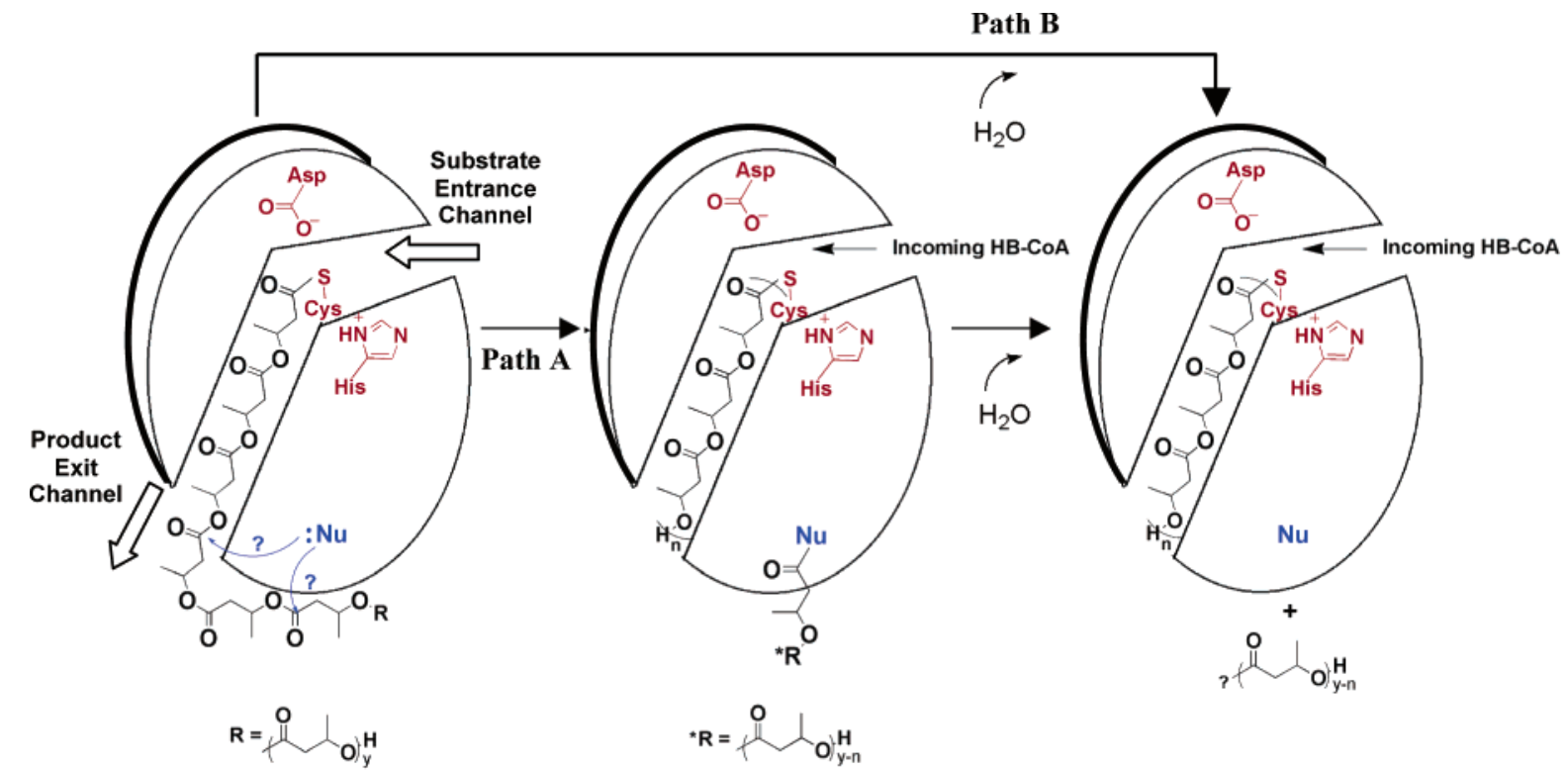

${ }^{a}$ Note that $\mathrm{Nu}$ can also represent a general-base catalyst.

recovery of radioactivity from the Sephadex G-50 column was $80 \%$, which corresponds to an $\mathrm{HB} / \mathrm{PhaCPhaE}_{\mathrm{Av}}$ ratio of 0.5 . The recovery of radioactivity from the C4-Vydac column was $84 \%$.

\section{RESULTS}

Evidence for Covalent Labeling of wt PhaCPhaE $E_{A v}$ by $\left[{ }^{14} \mathrm{C}\right] H B-C o A$. In our recent paper we reported that incubation of D302A-PhaCPhaE $\mathrm{Av}_{\mathrm{Av}}$ with $\left[{ }^{14} \mathrm{C}\right] \mathrm{HB}-\mathrm{CoA}$ resulted in detection, for the first time, of oligomeric $(\mathrm{HB})_{n}(n=3-10)$ covalently bound to $\mathrm{C} 149$ in $\mathrm{PhaC}_{\mathrm{Av}}$. The $(\mathrm{HB})_{n}-\mathrm{PhaC}_{\mathrm{Av}}$ could be chased into longer HB oligomers, suggesting that these species were intermediates in the polymerization process. Given our success with this mutant and the lessons learned, we focused on the detection of $(\mathrm{HB})_{n}-\mathrm{PhaC}_{\mathrm{Av}}$ using wt synthase. Incubation of $\left[{ }^{14} \mathrm{C}\right] \mathrm{HB}-\mathrm{CoA}$ with $\mathrm{PhaCPhaE} \mathrm{Av}_{\mathrm{Av}}$ at $\mathrm{S} / \mathrm{E}$ ratios of 5/1, 15/1, and 45/1 resulted in four distinct groups of products when analyzed by SDS-PAGE gels and autoradiography. These four species are numbered I-IV in Figure 1A; each species is composed of multiple products. No signals were detected on the phosphorimage when a similar experiment was carried out with C149A-PhaCPhaE $\mathrm{Av}_{\mathrm{Av}}$ and $\left[{ }^{14} \mathrm{C}\right] \mathrm{HB}-\mathrm{CoA}$, indicating that all observed signals result from covalently modified $\mathrm{PhaC}_{\mathrm{Av}}$, presumably through C149. The presence of four distinct groups of products supports previous observations that $\mathrm{PhaCPhaE}_{\mathrm{Av}}$ exhibits nonuniform loading of substrate at low S/E ratios in vitro (15). In fact, most of the synthase remains at the monomeric position of $\mathrm{PhaC}_{\mathrm{Av}}$ on the gel (labeled "position of PhaC" $(\sim 39 \mathrm{kDa})$, Figure 1A) in the region labeled species I, as determined by Coomassie staining (data not shown) and Western blot with Abs to PhaCPhaE $\mathrm{Av}_{\mathrm{Av}}$ (Figure 1B). A small amount of protein can be detected within the region of species II-IV if the Western blot is exposed for hours or the amount of reaction mixture analyzed by the gel is increased. In addition, since our Abs recognize both $\mathrm{PhaC}_{\mathrm{Av}}$ and $\mathrm{PhaE}_{\mathrm{Av}}$, it is unclear if species III and IV contain $\mathrm{PhaCPhaE}_{\mathrm{Av}}$ (not necessarily in a $1 / 1$ ratio) or $\mathrm{PhaC}_{\mathrm{Av}}$ alone. It is unlikely that species II contains $\mathrm{PhaE}_{\mathrm{Av}}$ because of the migration properties.

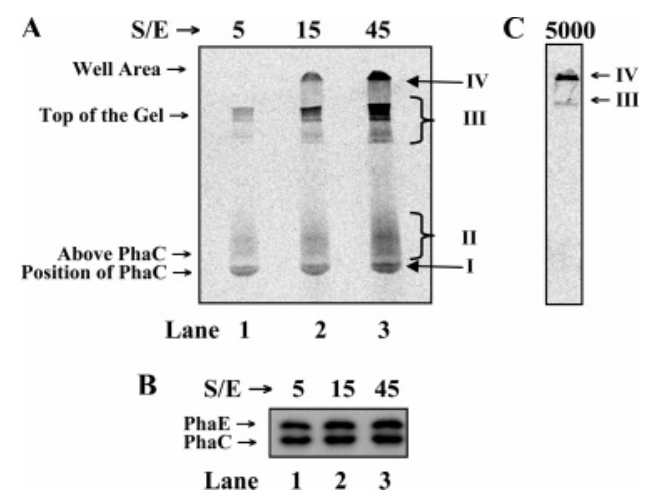

FIGURE 1: SDS-PAGE gel $(10 \%)$ analyzed by phosphorimaging (A, C) and Western blotting (B). The reactions contained $3.3 \mu \mathrm{M}$ PhaCPhaE $_{\mathrm{Av}}$ and $\left[{ }^{14} \mathrm{C}\right] \mathrm{HB}-\mathrm{CoA}\left(\mathrm{SA}\right.$ of $\left.2.6 \times 10^{7} \mathrm{cpm} / \mu \mathrm{mol}\right)$ at increasing $\mathrm{S} / \mathrm{E}$ ratios. All reactions were incubated for $5 \mathrm{~min}$ before examination. (A) Lanes $1-3$ correspond to $S / E$ ratios of 5, 15, and 45 , respectively. Species III but not species IV is able to enter the stacking gel. (B) Western blot of the same reaction mixtures described in $(\mathrm{A})$ revealing that most of the protein remains at the position of the $\mathrm{PhaC}_{\mathrm{Av}}$ monomer. (C) Phosphorimage demonstrating the chemical competence of species I and II at an S/E ratio of 5000. The SA of the substrate was $2.4 \times 10^{5} \mathrm{cpm} / \mu \mathrm{mol}$.

Species I is believed to be similar to the "intermediate species" $(\mathrm{HB})_{n}-\mathrm{PhaC}_{\mathrm{Av}}(n=3-10)$ recently identified and isolated using the $\mathrm{D} 302 \mathrm{~A}-\mathrm{PhaCPhaE} \mathrm{E}_{\mathrm{Av}}$ in a similar set of experiments (15). Lane 1 in Figure $1 \mathrm{~A}$ shows that at an S/E ratio of $5 / 1$, species I is the dominant product. In an effort to maximize our chances of isolating and identifying species I, we lowered the S/E ratio to $1 / 1$ and carried out trypsin digestion and HPLC analysis as described previously (15). The data revealed that peptides containing radioactivity eluted at acetonitrile concentrations $>62 \%$ (data not shown). Difficulties were encountered in identifying their composition as all fractions contained more than one peptide, with the exception of one which gave clean $\mathrm{N}$-terminal sequencing results. This fraction contained the same peptide that we had identified previously in studies with D302A-PhaCPhaE $\mathrm{Av}_{\mathrm{Av}}$ incubated with HB-CoA (15). ESI-MS analysis of this fraction gave a single peak with an $\left[\mathrm{M}+\mathrm{H}^{+}\right]$mass of 6039.0 


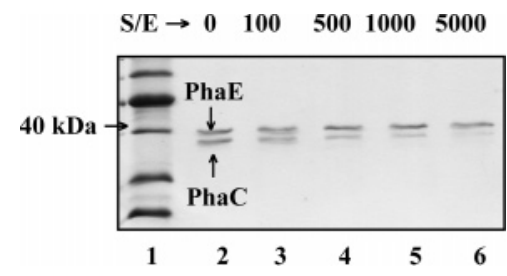

FIGURE 2: The Coomassie-stained SDS-PAGE gel shows disappearance of $\mathrm{PhaC}_{\mathrm{Av}}$ as the $\mathrm{S} / \mathrm{E}$ ratio increases. [PhaCPhaE $\mathrm{Ev}_{\mathrm{Av}}$ ] = $0.37 \mu \mathrm{M}$. Key: lane 1, MW standards; lanes 2-6 have S/E ratios of $0,100,500,1000$, and 5000, respectively. Unlabeled $(R)$-HB$\mathrm{CoA}$ was used in this experiment.

$\mathrm{Da}$; this mass corresponds to the molecular weight of the 52-mer peptide containing the active site cysteine attached with (HB) $)_{3}$, minus the MW of water (data not shown). Thus, similar $(\mathrm{HB})_{n}-\mathrm{PhaC}_{\mathrm{Av}}$ 's are generated with the wt synthase, but as noted earlier the nonuniform substrate loading hindered their identification.

Product Distribution When the S/E Ratio Is Changed. The results in Figure 1A indicated that, as the S/E ratio increases, the distribution of radiolabeled species (I-IV) changes. Our hypothesis was that species I-III might represent different stages of the synthase during the polymerization process and that the transition from one stage to the next could be mechanistically informative. Species IV, which does not enter the gel, is likely associated with long polymer chains that become aggregated due to our inability to control granule formation in vitro. Species IV is thus not considered further. Species II is thought to be a monomeric $\mathrm{PhaC}_{\mathrm{Av}}$ associated with $\mathrm{HB}$ oligomers longer than those associated with $\mathrm{PhaC}_{\mathrm{Av}}$ in species I. Species III resides way above the highest MW standard marker (175 kDa), and is able to migrate into the gel. Since recombinant $\mathrm{PhaCPhaE}_{\mathrm{Av}}$ in its native state is tetrameric $(\mathrm{MW}=320 \mathrm{kDa})$, species III could be either $(\mathrm{HB})_{n}-\mathrm{PhaC}_{\mathrm{Av}}$, where $n$ is much greater than $n$ of species II or $(\mathrm{HB})_{n}-\left(\mathrm{PhaCPhaE}_{\mathrm{Av}}\right)_{4}$. In the former case, $n$ would have to be $\geq 1600$, a less likely scenario as the polymer of this size would probably not be able to migrate into the gel. In the latter case, perhaps as the HB oligomer chain reaches a certain length, it is able to increase the stability of the tetrameric synthase/polymer complex such that it now survives the gel electrophoresis process. Abs to $\mathrm{PhaE}_{\mathrm{Av}}$ alone, presently not available, should distinguish between these options.

Our recent studies with D302A-PhaCPhaE $\mathrm{Av}_{\mathrm{v}}$ strongly suggested that species I is a self-primed synthase (15). To demonstrate the chemical competence of species I with the wt synthase, a reaction was performed at an S/E ratio of 5000/1. The results are shown in Figure 1C. At this S/E ratio, species I and II are no longer observed, and most of species III has disappeared. Only species IV (aggregated polymer) is observed in significant amounts. One could argue that because the SA of HB-CoA was lower than that in the reactions shown in Figure 1A, the radioactive signals in the regions of species II and III might not have been detected after just $\sim 15 \mathrm{~h}$ of exposure to the phosphorimager screen. Therefore, another experiment was carried out to monitor the disappearance of $\mathrm{PhaC}_{\mathrm{Av}}$ by Coomassie staining as a function of $\mathrm{S} / \mathrm{E}$ ratio. As shown in Figure 2, $\mathrm{PhaC}_{\mathrm{Av}}$ gradually disappears from the monomeric position, and presumably is distributed at other positions on the gel. In fact, when a higher concentration of synthase and S/E ratios greater than 500

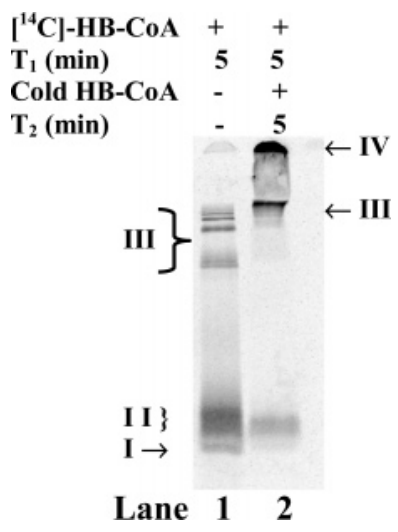

FIGURE 3: Chemical competence of species I-III in PHB Formation. A reaction containing $3.3 \mu \mathrm{M}$ PhaCPhaE $\mathrm{Av}_{\mathrm{Av}}$ and $148 \mu \mathrm{M}\left[{ }^{14} \mathrm{C}\right]-$ $\mathrm{HB}-\mathrm{CoA}(\mathrm{S} / \mathrm{E}=45)$ was prepared in duplicate. After 5 min of incubation $\left(T_{1}\right)$, one reaction was quenched with Laemmli buffer (lane 1), while additional cold HB-CoA was added to the other at an S/E ratio of 1000 . The reaction was allowed to proceed for another $5 \min \left(T_{2}\right)$ before being quenched (lane 2). Autoradiography of the gel is shown.

(data not shown) are used in the reactions and loaded onto the gel, protein appears near the top of the gel and in the well area where species III and IV, respectively, reside. This result also suggests that only in the presence of high concentrations of HB-CoA relative to the synthase is all of the $\mathrm{PhaC}_{\mathrm{Av}}$ modified. Interestingly, the migratory properties of $\mathrm{PhaE}_{\mathrm{Av}}$ do not appear to change in any of these reactions. Again, it is unknown whether a small amount of $\mathrm{PhaE}_{\mathrm{Av}}$ is associated with species III and IV. It is also possible that the interaction between $\mathrm{PhaE}_{\mathrm{Av}}$ and the PHB polymer is weakened in SDS.

To determine if the synthase can add substrate onto preexisting $\mathrm{HB}$ oligomers or polymer in vitro, implying a nonprocessive polymerization mechanism, radiolabeled species I, formed at an S/E ratio of 45/1 (lane 1 in Figure 3), was chased with cold HB-CoA at an S/E ratio of 1000/1. As shown in Figure 3, most of the radioactivity was associated with species III and IV (lane 2). This result demonstrates that the transition from species I to species II, III, or IV is nonprocessive since cold HB-CoA is directly added onto the preformed $\left[{ }^{14} \mathrm{C}\right] \mathrm{HB}$ oligomers to form longer oligomers. In addition, the results suggest that species I contains intermediates on the pathway of PHB polymer formation. In fact, species I-III observed in lane 1 (Figure 3) are all chemically competent, since their intensities decreased significantly (Figure 3, lane 2).

Despite the fact that the same amount of radioactivity was used in reactions shown in lanes 1 and 2 of Figure 3, quantitative analysis reveals that the amount of radioactivity detected in lane 2 is $\sim 2-3$-fold higher than that in lane 1 . This result suggests that short $(\mathrm{HB})_{n}-\mathrm{PhaC}(n=1-3)$ formed at low S/E ratios is prone to hydrolysis during gel electrophoresis as we have previously shown with the sT-CoA studies (14). This process is less likely to occur as the HB oligomer chain extends.

Kinetics of the Polymerization Reaction Catalyzed by wt PhaCPhaE $E_{A v}$ Monitored by Anti-PhaCPhaE $E_{A v}$ Western Blot: Evidence for Polymer Reinitiation. The reactions described thus far contain micromolar concentrations of synthase and low S/E ratios. Due to the high turnover number of the enzyme, only the end products of the reactions have thus 
A
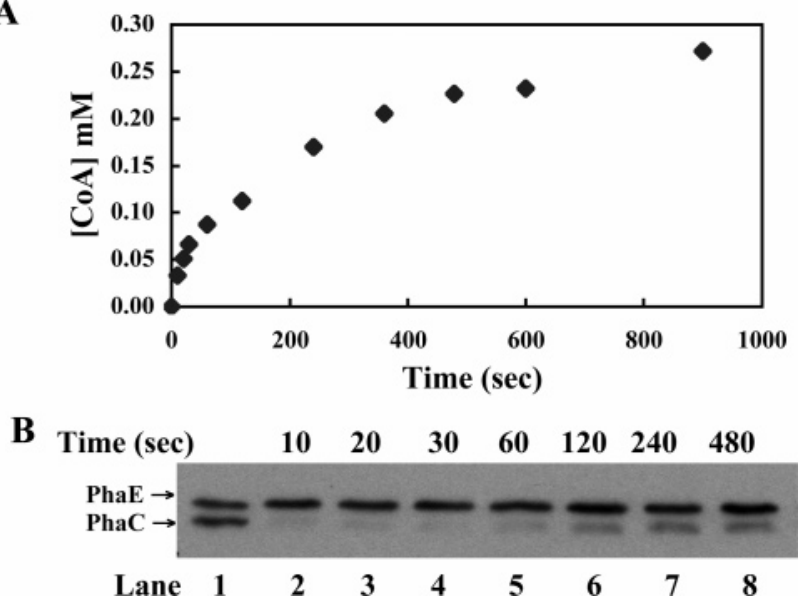

Figure 4: Kinetics of wt $\mathrm{PhaCPhaE}_{\mathrm{Av}}$ monitored by CoA release (A) and a Western blot using $\mathrm{Abs}$ to PhaCPhaE $\mathrm{Pv}_{\mathrm{Av}}$ (B). (A) In a final volume of $80 \mu \mathrm{L}, 10 \mathrm{nM}$ PhaCPhaE $\mathrm{Av}_{\mathrm{Av}}$ was reacted with 0.7 $\mathrm{mM}$ cold HB-CoA $(\mathrm{S} / \mathrm{E}=70000)$ in assay buffer at RT. (B) At $10,20,30,60,120,240$, and 480 s (lanes $2-8$, respectively), 10 $\mu \mathrm{L}$ was removed from the reaction mixture described in $(\mathrm{A})$ and quenched with an equal volume of Laemmli buffer. From this quenched mixture, $\sim 7.2 \mathrm{ng}$ of $\mathrm{PhaCPhaE}_{\mathrm{Av}}$ was loaded onto the gel and resolved, followed by transfer to a PVDF membrane and probing with $\mathrm{PhaCPhaE}_{\mathrm{Av}}$ Abs. Lane 1 contains $7.2 \mathrm{ng}$ of $\mathrm{PhaCPhaE}_{\mathrm{Av}}$ only.

far been analyzed. In an effort to better understand the in vitro polymerization, we lowered the $\mathrm{PhaCPhaE}_{\mathrm{Av}}$ concentration to $10 \mathrm{nM}$ so that kinetics by CoA release could be monitored. We also monitored the fate of the protein by Western blotting using Abs to $\mathrm{PhaCPhaE}_{\mathrm{Av}}$. The $\mathrm{S} / \mathrm{E}$ ratio was increased to 70000. Autoradiography can no longer be used to monitor the formation of ${ }^{14} \mathrm{C}$-labeled species I and II under these conditions as $\left[{ }^{14} \mathrm{C}\right] \mathrm{HB}-\mathrm{CoA}$ with an $\mathrm{SA}$ of $10^{10} \mathrm{cpm} / \mu \mathrm{mol}$ is not available and would be required for phosphorimager detection. A typical profile showing the multiple phases of CoA release is shown in Figure 4A. Concomitantly monitoring the same reaction by Western analysis gave the surprising results shown in Figure 4B. Within the first $10 \mathrm{~s}$ of the reaction, all of the $\mathrm{PhaC}_{\mathrm{Av}}$ had disappeared. This was anticipated since results from Figure 2 have already demonstrated that $\mathrm{PhaC}_{\mathrm{Av}}$ incubated with $\mathrm{HB}$ $\mathrm{CoA}$ at $\mathrm{S} / \mathrm{E} \geq 5000$ migrated near the top of the gel or did not enter the gel. However, as the reaction proceeded, $\mathrm{PhaC}_{\mathrm{Av}}$ gradually reappeared as shown in lanes 6-8 (Figure 4B), which contain reactions that were quenched after 120, 240, and $480 \mathrm{~s}$, respectively. Where does this $\mathrm{PhaC}_{\mathrm{Av}}$ come from? The CoA release assay (Figure 4A) shows that, at $120 \mathrm{~s}$, approximately $16 \%$ of the substrate has been consumed. This corresponds to an $\mathrm{S} / \mathrm{E}$ ratio of 22400 (on the basis of one PHB chain per dimer PhaCPhaE $\mathrm{Av}_{\mathrm{Av}}(14)$ ), indicating that the molecular weight of the polymer formed is $1.8 \mathrm{MDa}$ if the synthase is uniformly loaded under these conditions. The molecular weight of a PHB polymer formed by $\mathrm{PhaCPhaE}_{\mathrm{Av}}$ in vitro has previously been determined by Jossek et al. to be $1.6 \times 10^{6} \mathrm{~g} / \mathrm{mol}(19)$. Thus, it is interesting that $\mathrm{PhaC}_{\mathrm{Av}}$ reappears once the synthesis of a $\mathrm{PHB}$ polymer chain is complete. The time period for $\mathrm{PhaC}_{\mathrm{Av}}$ reappearance appears to be correlated with the initial burst phase of CoA release. Analysis of a $\mathrm{CoA}$ release profile with $18 \mathrm{nM}$ synthase at an $\mathrm{S} / \mathrm{E}$ ratio of 55000/1 gave similar results (data not shown). As discussed subsequently, this result suggests that $\mathrm{PhaC}_{\mathrm{Av}}$ can catalyze its own reinitiation.

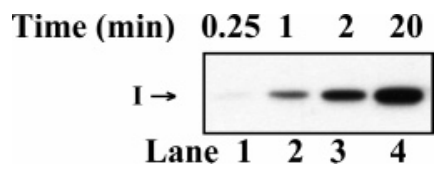

FIGURE 5: Western blot using PHB Abs showing the time dependence associated with species I after complete consumption of $\mathrm{HB}-\mathrm{CoA}$. PhaCPhaE $\mathrm{Av}_{\mathrm{Av}}, 1.25 \mu \mathrm{M}$, was incubated with $18.75 \mu \mathrm{M}$ unlabeled $(R)-\mathrm{HB}-\mathrm{CoA}(\mathrm{S} / \mathrm{E}=15)$ in a final volume of $70 \mu \mathrm{L}$ at $30{ }^{\circ} \mathrm{C}$. At this concentration of synthase, all of the substrate is consumed in less than milliseconds. At 0.25, 1, 2, and 20 min (lanes $1-4$, respectively), $15 \mu \mathrm{L}$ was removed from the reaction mixture and quenched with an equal volume of Laemmli buffer without reducing reagent. All reaction products were resolved by SDSPAGE, transferred onto a PVDF membrane, and probed with PHB Abs. Each lane contains equal amounts of protein $(\sim 1.5 \mu \mathrm{g})$.

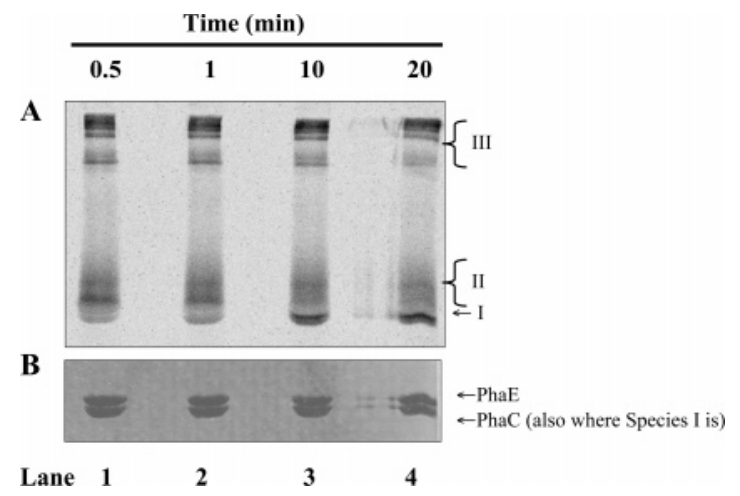

FIGURE 6: Time-dependent phenomenon observed with wt $\mathrm{PhaCPhaE}_{\mathrm{Av}}$ and $\left[{ }^{14} \mathrm{C}\right] \mathrm{HB}-\mathrm{CoA}$. In a final volume of $45 \mu \mathrm{L}, 3.5$ $\mu \mathrm{M}$ PhaCPhaE ${ }_{\mathrm{Av}}$ was reacted with $158 \mu \mathrm{M}\left[{ }^{14} \mathrm{C}\right] \mathrm{HB}-\mathrm{CoA}(\mathrm{S} / \mathrm{E}=$ 45 ) in assay buffer. At $0.5,1,10$, and $20 \mathrm{~min}, 10 \mu \mathrm{L}$ of the reaction mixture was removed and quenched with equal volumes of Laemmli buffer without reducing reagent. The reaction products were resolved on an SDS-PAGE gel. Key: (A) autoradiography; (B) part of the Coomassie-stained gel used in (A).

Time-Dependent Phenomenon at Low S/E Ratios: Evidence for Premature Termination. The polymerization reaction at low $\mathrm{S} / \mathrm{E}$ ratios has also been investigated further due to unexpected observations. Under the experimental conditions described in Figure 1A, 3.3 $\mu \mathrm{M}$ synthase, which has an SA of $\sim 200$ units $/ \mathrm{mg}$, consumes all the $\left[{ }^{14} \mathrm{C}\right] \mathrm{HB}-\mathrm{CoA}$ (16.5-148.5 $\mu \mathrm{M})$ within milliseconds. Serendipitously, during several experiments we examined the products by SDSPAGE at various times after complete substrate consumption. An example of what we will subsequently call "a timedependent phenomenon" is shown in Figure 5. In this experiment, the products were examined by SDS-PAGE after complete HB-CoA consumption at 0.25-20 min, followed by transfer to a PVDF membrane and analysis with PHB Abs. The observed species migrate in the region of monomeric $\mathrm{PhaC}_{\mathrm{Av}}$ on the gel, and with time, an increase in the amount of species I is observed. We have determined that the PHB Abs can detect HB oligomers within species I and they do not cross-react with $\mathrm{PhaCPhaE}_{\mathrm{Av}}$ (data not shown). Several interpretations of this experiment are possible. One is that the $(\mathrm{HB})_{n}$ units from $\mathrm{PhaC}_{\mathrm{Av}}$ are "transferred" to apo-PhaC $\mathrm{Av}_{\mathrm{Av}}$. A second interpretation is that $(\mathrm{HB})_{n^{-}}$ $\mathrm{PhaC}_{\mathrm{Av}}$ 's ( $n$ is low, $=3-10$ or slightly longer) are regenerated directly.

To confirm the time-dependent observation with PHB Abs, a similar reaction was carried out with $\left[{ }^{14} \mathrm{C}\right] \mathrm{HB}-\mathrm{CoA}$ and monitored using autoradiography (Figure 6A). The intensity of species I increased in lanes $1-4$ and that of species II 


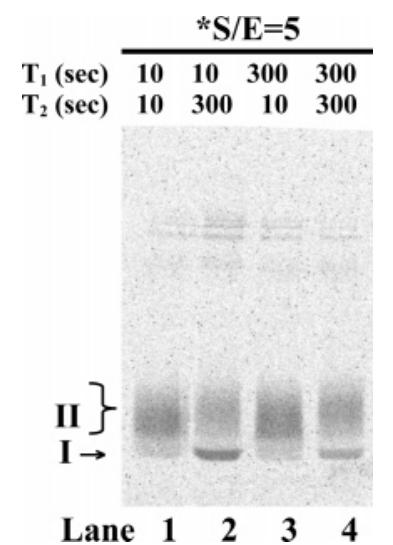

FIGURE 7: Autoradiography revealing the reactivity of species I and II, and the basis for the time-dependence phenomenon. A reaction containing $3.3 \mu \mathrm{M}$ PhaCPhaE ${ }_{\mathrm{Av}}$ and $16.5 \mu \mathrm{M}$ cold $\mathrm{HB}$ $\mathrm{CoA}(\mathrm{S} / \mathrm{E}=5)$ was prepared in quadruplicate and treated as follows: lanes 1 and 2, additional $\left[{ }^{14} \mathrm{C}\right] \mathrm{HB}-\mathrm{CoA}$ (SA of $3.3 \times 10^{7}$ $\mathrm{cpm} / \mu \mathrm{mol}$ ) was added after $10 \mathrm{~s}$ and allowed to react for either 10 or $300 \mathrm{~s}$, respectively, before quenching with Laemmli buffer; lanes 3 and 4, additional $\left[{ }^{14} \mathrm{C}\right] \mathrm{HB}-\mathrm{CoA}$ was added after $300 \mathrm{~s}$ and allowed to react for either 10 or $300 \mathrm{~s}$ before being quenched. The amount of $\left[{ }^{14} \mathrm{C}\right] \mathrm{HB}-\mathrm{CoA}$ added to each reaction was exactly the same, at a $\left[{ }^{14} \mathrm{C}\right] \mathrm{HB}-\mathrm{CoA} / \mathrm{PhaCPhaE} \mathrm{Av}_{\mathrm{Av}}$ ratio of $5 . T_{1}$ and $T_{2}$ refer to the reaction times with cold and hot substrate, respectively.

gradually decreased as analyzed by quantitative analysis using ImageQuant TL software. Even more perplexing was the observation shown in Figure 6B that the amount of protein in the region of the gel where species I resides does not appreciably change as a function of time. This result was later confirmed with the more sensitive Western analysis using PhaCPhaE $\mathrm{Av}_{\mathrm{Av}} \mathrm{Abs}$ (data not shown). The results suggest that material from region II (HB) ${ }_{n}-\mathrm{PhaC}_{\mathrm{Av}}(n>40$ and $<$ 100 on the basis of the migratory properties of D302A$\mathrm{PhaC}_{\mathrm{Av}} / \mathrm{HB}$ oligomer complexes at $\mathrm{S} / \mathrm{E}$ ratios up to $100 / 1$, (15)) is being converted into material in region I $(\mathrm{HB})_{n^{-}}$ $\mathrm{PhaC}_{\mathrm{Av}}(n \geq 3-10)$. Although the reaction is very slow, we believe it is mechanistically informative. One interpretation of these observations is that the PHB chain is being transferred to a second amino acid residue on the protein which is then hydrolyzed and lost, leaving behind species I, a "primed synthase" (Scheme 1).

Probing the Reactivity of Species I and II Reveals the Basis of the Time-Dependent Phenomenon. Information on the reactivity of species I and II was obtained by monitoring the distribution of radiolabel as a small amount of $\left[{ }^{14} \mathrm{C}\right] \mathrm{HB}-$ $\mathrm{CoA}$ is introduced into the reaction mixture that contains species I and II which are preformed with unlabeled HBCoA. Results from this study are shown in Figure 7. To reactions containing synthase preincubated with a 5-fold excess of cold HB-CoA for 10 or $300 \mathrm{~s}$ was added $\left[{ }^{14} \mathrm{C}\right]-$ HB-CoA at an S/E ratio of 5/1, enough to be detectable by phosphorimager. The final products after 10 or $300 \mathrm{~s}$ of incubation were analyzed by SDS-PAGE. Reactions with cold substrate at an S/E ratio of 5 allowed examination of species I and II.

Lane 1 of Figure 7 reveals that almost all of the radiolabel is associated with species II after the synthase is reacted with cold HB-CoA (S/E ratio of 5) for $10 \mathrm{~s}$, followed by addition of $\left[{ }^{14} \mathrm{C}\right] \mathrm{HB}-\mathrm{CoA}$ and incubation for an additional $10 \mathrm{~s}$. When the period of incubation with the labeled substrate is extended to $300 \mathrm{~s}$, however, the time-dependent phenomenon of
Table 1: Quantitation of Autoradiography Signals in Lanes 1 and 2 of Figure $7^{a}$

\begin{tabular}{lrrc}
\hline & lane 1 & lane 2 & lane 2- lane 1 \\
\hline species I & 18081 & 86687 & +68605 \\
species II & 259587 & 137503 & -122084 \\
total & 280795 & 227316 & -53479 \\
\hline
\end{tabular}

${ }^{a}$ The numbers are reported as volume intensity.

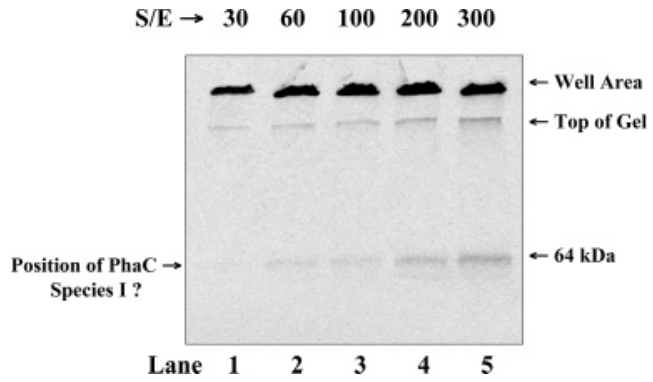

FIGURE 8: Autoradiography of products from reactions containing recombinant $\mathrm{PhaC}_{\mathrm{We}}$ and $\left[{ }^{14} \mathrm{C}\right] \mathrm{HB}-\mathrm{CoA}\left(\mathrm{SA}\right.$ of $7.9 \times 10^{6} \mathrm{cpm} /$ $\mu \mathrm{mol}$ ) at various $\mathrm{S} / \mathrm{E}$ ratios. [ $\left.\mathrm{PhaC}_{\mathrm{We}}\right]=3 \mu \mathrm{M}$. For lanes $1-5, \mathrm{~S} / \mathrm{E}$ $=30,60,100,200$, and 300, respectively. The phosphorimage was obtained after 2 days of exposure to the low-energy screen.

species I is once again observed (lane 2, Figure 7). However, it is now clear that the additional formation of species I after all of the initial substrate is consumed is a result of modification of species II. In fact, the $\mathrm{PhaC}_{\mathrm{Av}}$ with $\mathrm{HB}$ units that have been most recently added onto species II is converted to species I. This result suggests that cleavage somewhere along the HB oligomer chain associated with species II has occurred. Quantitative analysis of the phosphorimage (Table 1) revealed that the observed signal intensity of species II in lane 2 is $50 \%$ of that in lane 1 (137503/259587), and the signal intensity of species I in lane 2 is 4.7-fold higher that that in lane $1(86687 / 18081)$. Overall, the total amount of the observed signal intensity in lane 2 is $20 \%$ less than that in lane $1(53479 / 280795)$, despite the fact that the same amount of radioactivity was used in both reactions. This observation suggested that the cleavage that occurred within the radiolabeled portion of the polymer chain during the extended reaction incubation time resulted in the loss of a portion of labeled polymer that is adjacent to the nonlabeled polymer during electrophoresis.

Lane 2 of Figure 7 provides a preview of product distribution in the reaction shown in lane 3 before $\left[{ }^{14} \mathrm{C}\right] \mathrm{HB}$ CoA was added (since the synthase in lane 3 was incubated with cold substrate for $300 \mathrm{~s}$ initially). Species I observed in lane 2 was almost completely gone in lane 3 , suggesting that species I is more reactive than or as reactive as species II. When incubation with labeled HB-CoA is extended to $300 \mathrm{~s}$, species I again returns as shown in lane 4, although the amount is less.

Labeling of Recombinant Class I Synthase PhaC $C_{W e}$. The observation of intermediate species in the reactions catalyzed by recombinant class III synthase $\mathrm{PhaCPhaE}_{\mathrm{Av}}$ led us to carry out a similar study on the recombinant class I synthase $\mathrm{PhaC}_{\mathrm{We}}$. Autoradiography results in Figure 8 establish by yet another method, that recombinant $\mathrm{PhaC}_{\mathrm{We}}$ does not exhibit uniform loading of the substrate (8). All radioactivity ends up in the well area of the gel at low S/E ratios (e.g., 30), while most of the synthase remains unmodified as revealed by Coomassie staining of the gel or by Western 
blots with $\mathrm{Abs}$ to $\mathrm{PhaC}_{\mathrm{We}}$ (data not shown). In fact, even at an S/E ratio of 5/1, the same results are observed (data not shown). However, at high enough S/E ratios (>200/1), small amounts of radioactivity are observed at the monomeric position of $\mathrm{PhaC}_{\mathrm{We}}$ (Figure 8, lanes 4 and 5). These species are proposed to be similar to species I observed with the class III PhaCPhaE $\mathrm{Av}_{\mathrm{Av}}$ system.

\section{DISCUSSION}

In the present paper, we report on our continuing efforts to understand the in vitro polymerization catalyzed by recombinant class III synthase $\mathrm{PhaCPhaE}_{\mathrm{Av}}$. Recently, we presented the first direct evidence using D302A-PhaCPhaE $\mathrm{Av}_{\mathrm{Av}}$, a mutant severely impaired in the elongation process, that HB oligomers ranging in length from trimers to decamers are covalently attached to the active site cysteine (C149) through a thioester linkage (15). The intermediate species detected with this mutant are believed to be the same as species I (Figure 1A) generated by interaction of HB-CoA with the wt synthase. In comparison to D302A-PhaCPhaE $\mathrm{Av}_{\mathrm{Av}}$ at low $\mathrm{S} / \mathrm{E}$ ratios $(<100)(15)$, it is clear that uniform loading of the wt synthase with HB-CoA is not occurring in the reaction at similar S/E ratios.

Our current studies further demonstrate that species I is chemically competent and that it represents intermediates in the synthesis of larger PHB polymers. Species I is only observed at low $\mathrm{S} / \mathrm{E}$ ratios when there is not enough substrate to form large polymers. Our model is that species I results from self-priming by the synthase. We have also shown that species II and III are chemically competent from the experiment in Figure 3. The disappearance of species I-III upon the addition of cold $\mathrm{HB}-\mathrm{Co}$ A to preformed $\left[{ }^{14} \mathrm{C}\right](\mathrm{HB})_{n^{-}}$ synthase complex indicates that species II and III can be chased into synthase with longer polymers attached. Our current hypothesis is that species II is $(\mathrm{HB})_{n}-\mathrm{PhaC}_{\mathrm{Av}}$, where $n$ is approximately $40-100$, on the basis of its migratory properties in the gel. We believe that species III is (HB) $n^{-}$ $\mathrm{PhaCPhaE}_{\mathrm{Av}}$, where $n$ is similar to or greater than that observed in species II and that the protein is in a tetrameric state stabilized by the $(\mathrm{HB})_{n}$ chain. The transition from species I to II to III may in part be governed by the length of the HB chain.

The most interesting results we have reported are the unexpected observations made on SDS-PAGE analysis of the products generated when the synthase was incubated at both high and low $\mathrm{S} / \mathrm{E}$ ratios. The reappearance of $\mathrm{PhaC}_{\mathrm{Av}}$ at its monomeric position in the gel after the completion of the synthesis of a PHB polymer with a molecular weight of $1.8 \mathrm{MDa}$ at high $\mathrm{S} / \mathrm{E}$ ratios (Figure $4 \mathrm{~A}$ ) and the timedependent observation of species I at low S/E ratios (Figure 6) have led us to propose that $\mathrm{PhaCPhaE}_{\mathrm{Av}}$ can catalyze PHB chain termination and repriming as shown in Scheme 1. This process can also be expressed as $y \mathrm{HB}-\mathrm{CoA}+\mathrm{E} \rightarrow \mathrm{E}-(\mathrm{HB})_{y}$ $\rightarrow \mathrm{E}-(\mathrm{HB})_{n}+(\mathrm{HB})_{y-n}$, where $\mathrm{E}$ is the synthase. In this scheme, the active form of the synthase during elongation is shown in the dimeric state, with the second monomer represented by the darker line and only one of the two monomers supporting a polymer chain. Our previous studies with sT-CoA have demonstrated that there is one PHB chain per dimer in both the class I and class III synthases (14, 20 ). In addition, Scheme 1 proposes that there are two channels within each monomer of synthase, an entrance channel for substrate binding and an exit channel for polymer egress. This model is based on the structure of the thioesterase domain of the surfactin synthase, an $\alpha / \beta$ hydrolase superfamily member that cyclizes a peptide through an ester bond formation using the hydroxyl group of a 3-(R)-hydroxyalkanoate (C13-C15 fatty acid) (21). This model is also based on the fact that lipases, also $\alpha / \beta$ hydrolase family members, have provided threading models for the PHB synthases (17, 22 ). At present, there is no member of this family that possesses a single active site formed at the interface of the two monomers. The active sites of these enzymes are also deeply buried (8). Thus, how one active site could be generated from two $\alpha / \beta$ hydrolase monomers as required in our original model for PHB synthase is difficult to reconcile with available structural information. An addition in this scheme, to accommodate the present results, is a nucleophile $(\mathrm{Nu})$ that is proposed to catalyze a chain-transfer (path A, Scheme 1) or a hydrolysis (path B, Scheme 1) reaction and regeneration of a primed synthase. In path $\mathrm{A}$, hydrolysis could occur once the polymer chain is transferred onto the putative amino acid residue. Alternatively, the amino acid could function as a general-base catalyst to activate a water (path B). A number of conserved amino acids of unknown function (D84, D177, D251, S90, Y81, or H303, $\mathrm{PhaC}_{\mathrm{Av}}$ numbering) are candidates for this nucleophile. Finally, the putative $\mathrm{Nu}$ directly or indirectly responsible for the chaintransfer step does not have to reside on the same synthase monomer as the active site cysteine. Although the active form of the synthase has been determined to be dimeric, one monomer could play a role in synthesis, while the other participates only in chain termination.

The studies of the polymerization reaction at high S/E ratio (70000) in vitro provide the most compelling evidence for this model. $\mathrm{PhaC}_{\mathrm{Av}}$, when incubated with substrate, rapidly disappears at first due to covalent attachment of the large PHB polymers. However, $(\mathrm{HB})_{n}-\mathrm{PhaC}_{\mathrm{Av}}(n \geq 3-10)$ then reappears (Figure 4B), a process that requires $\mathrm{PhaC}_{\mathrm{Av}}$ to become detached from the bulk of the polymer. The reappearance seems to be correlated with the polymer size $\left(1.8 \times 10^{6} \mathrm{Da}\right)$. The slow phase of CoA release would then be correlated with hydrolysis of the bulk polymer and conformational reorientation such that a new polymer can be generated from the remaining primed synthase. This model is supported by the work of Jossek et al., who carried out studies of granule formation by $\mathrm{PhaCPhaE}_{\mathrm{Av}}$ in vitro. At a substrate concentration of $13.4 \mathrm{mM}$ and synthase concentration of $\mathrm{nM}$, PhaCPhaE $\mathrm{Av}_{\mathrm{Av}}$ was shown to generate $\sim 25$ polyester chains, with a mass of $\sim 1.6 \times 10^{6} \mathrm{~g} / \mathrm{mol}$ (19). No model for this reinitiation was proposed.

Finally, these results are in agreement with our recent in vivo studies of $W$. eutropha under transient PHB formation and PHB accumulation conditions (23). These studies demonstrated that, with the class I synthase, each $\mathrm{PhaC}_{\mathrm{We}}$ has been reused $\sim 60$ times in the former case and $\sim 200$ times in the latter case to account for the amount of $\mathrm{PhaC}_{\mathrm{We}}$ and PHB within the cell and the size of the polymer. Therefore, the ability of both classes of synthases to reinitiate is a common theme observed in vivo or in vitro. Our data suggest a model for this process, very different from the model in which the thioester in the active site is hydrolyzed directly to leave apo-PhaC $(24,25)$. 
The study of the time-dependent reappearance of $\mathrm{PhaC}_{\mathrm{Av}}$ observed at low $\mathrm{S} / \mathrm{E}$ ratios is unlikely to actually occur in vivo (Figure 7). However, it provides further support for Scheme 1 and also insight regarding the site of cleavage within the polymer chain. The study was designed such that a "cold" portion of polymer was generated first, followed by a "hot" portion which is closer to the active site of the synthase. When the reaction with $\left[{ }^{14} \mathrm{C}\right] \mathrm{HB}-\mathrm{CoA}$ is only allowed to proceed for $10 \mathrm{~s}$, we see that all of the radioactivity is associated with species II (lane 1, Figure 7). Assuming $\sim 5 \%$ of the $\mathrm{PhaC}_{\mathrm{Av}}$, estimated by Western analyses, is associated with species II in lane 1, the average length of $\mathrm{HB}$ oligomers attached to $\mathrm{PhaC}_{\mathrm{Av}}$ can be calculated to be 40-100 units. However, when this reaction is left sitting for $5 \mathrm{~min}$, a portion of the radioactivity is observed to be associated with species I (compare lanes 1 and 2 in Figure 7), indicating a portion of species II has undergone modification to become species I. Scheme 1 provides a rationale for this observation. In the absence of additional HB-CoA, the oligomer associated with species II could also be subject to attack by the $\mathrm{Nu}$ (Scheme 1) that is positioned to catalyze the chain-transfer step. This process is very slow in comparison with that of the enzyme that is loaded with very large polymer. If the oligomer chain cleavage occurs closer to the active site of the synthase, somewhere along the radiolabeled portion of the $\mathrm{HB}$ oligomer, Scheme 1 predicts the loss of total recoverable radioactivity as the $\mathrm{HB}$ oligomer (some of which is radiolabeled) associated with $\mathrm{Nu}$ is eventually removed through hydrolysis and consequently is not recovered by SDS-PAGE. Quantitative analysis of the phosphorimage confirms this prediction (Table 1 ). The amount of total radioactivity recovered in lane 2 is approximately $20 \%$ less than that in lane 1 , despite the fact that the same amount of radioactivity was used in both reactions. Comparison of the signals of species II in lanes 1 and 2 revealed that half of species II in lane 1 had undergone the transition to form species I. Of this half, $\sim 44 \%$ of the radioactivity was lost during the transition (53479/122084, Table 1), while the rest (68605) remained associated with $\mathrm{PhaC}_{\mathrm{Av}}$. If as little as $2.5 \%$ of species II in the form of (HB) ${ }_{40}-\mathrm{PhaC}_{\mathrm{Av}}$ undergoes transition to form species I as (HB) ${ }_{20}-\mathrm{PhaC}_{\mathrm{Av}}$, then the amount of radiolabel increase in species I from lane 1 to lane 2 can be accounted for. In this scenario, the amount of $\mathrm{PhaC}_{\mathrm{Av}}$ remaining at the monomeric position $(\sim 39 \mathrm{kDa})$ in lanes 1 and 2 would only increase from $95 \%$ to $\sim 97.5 \%$. This difference is not differentiable by Coomassie staining and Western analysis (Figure 6). Therefore, the increasing amounts of species I observed as a function of time in Figures 5-7 most likely resulted from species II whose HB oligomers were cleaved at approximately $20 \mathrm{HB}$ units away from the active site C149. The synthase with a 20 -mer $\mathrm{HB}$ oligomer is less prone to hydrolysis catalyzed by D302 than very short oligomers (15), and thus, this product (a member of species I) is observed to increase over time.

We believe that primed synthase is also observed with recombinant class $\mathrm{I} \mathrm{PhaC}_{\mathrm{We}}$ (Figure 8 ). However, the study of this synthase is complicated by the fact that, in vitro, its elongation rate is so much greater than the initiation rate that most of the synthase remains untouched at low S/E ratios. From a comparison of the autoradiographs in Figures 1 and 8 , it is clear that wt $\mathrm{PhaCPhaE}_{\mathrm{Av}}$ loads substrate more uniformly than $\mathrm{PhaC}_{\mathrm{We}}$ at low $\mathrm{S} / \mathrm{E}$ ratios. These results suggest that the polymerization reaction catalyzed by the recombinant $\mathrm{PhaC}_{\mathrm{We}}$ in vitro system may be missing a factor or factors, perhaps a $\mathrm{PhaE}_{\mathrm{Av}}$ equivalent. We attempted to isolate the native $\mathrm{PhaC}_{\mathrm{We}}$ from its host by using a mutant $W$. eutropha strain in which the synthase gene was replaced with the same gene containing a (His) 6 tag attached at its $\mathrm{N}$-terminus. Despite the fact that this same construct in Escherichia coli resulted in rapid synthase isolation in high yield (16), the tag did not facilitate its isolation from $W$. eutropha. Using many chromatographic steps, we have purified the synthase to $\sim 20 \%$ homogeneity (data not shown). Interestingly, the kinetics of CoA release of this "protein mixture" differs from that of recombinant $\mathrm{PhaC}_{\mathrm{We}}$ in that the lag phase for CoA release (8) is no longer observed. In fact, the kinetics now resembles that for $\mathrm{PhaCPhaE}_{\mathrm{Av}}$ in Figure 4. The discrepancy observed is a clear indication that either the native and recombinant class I synthase are different or the "protein mixture" contains factor(s) that are important in initiation.

To summarize, we have demonstrated that class III $\mathrm{PhaCPhaE}_{\mathrm{Av}}$ synthase initiates polymerization through selfpriming. The primed synthase, species I, can serve as a substrate for further PHB elongation. Since HB units can be added onto the existing polymers when $\mathrm{HB}-\mathrm{CoA}$ is introduced into a reaction mixture containing preformed intermediate species, the reaction catalyzed by $\mathrm{PhaCPhaE}_{\mathrm{Av}}$ is nonprocessive. The caveat is that we have only observed species I-III at low S/E ratios. Therefore, when enough HB$\mathrm{CoA}$ (S/E ratio is high) is available to load all of the enzyme present, the polymerization may be processive such that no intermediates can be detected and preformed oligomers cannot be extended, similar to the observations made with the enzyme that makes polyphosphates, polyphosphate kinase (26). Our studies suggest that the synthase also has a hydrolase activity that allows the synthase itself to control the polymer size and reinitiation. Further investigations are necessary to obtain support for the model in Scheme 1.

\section{ACKNOWLEDGMENT}

We thank Dr. Gregory York for the construction of the W. eutropha gene replacement strain containing (His) ${ }_{6}-\mathrm{PhaC}$.

\section{REFERENCES}

1. Byrom, D. (1987) Polymer Synthesis by Microorganisms: Technology and Economics, Trends Biotechnol. 5, 246-250.

2. Anderson, A. J. and Dawes, E. A. (1990) Occurrence, Metabolism, Metabolic Role, and Industrial Uses of Bacterial Polyhydroxyalkanoates, Microbiol. Rev. 54, 450-472.

3. Madison, L. L. and Huisman, G. W. (1999) Metabolic engineering of poly(3-hydroxyalkanoates): From DNA to plastic, Microbiol. Mol. Biol. Rev. 63, 21-53.

4. Rehm, B. H. and Steinbüchel, A. (1999) Biochemical and genetic analysis of PHA synthases and other proteins required for PHA synthesis, Int. J. Biol. Macromol. 25, 3-19.

5. Gerngross, T. U. (1999) Can biotechnology move us toward a sustainable society? Nat. Biotechnol. 17, 541-544.

6. Rehm, B. H. (2003) Polyester synthases: natural catalysts for plastics, Biochem. J. 376, 15-33.

7. Stubbe, J. and Tian, J. (2003) Polyhydroxyalkanoate (PHA) homeostasis: the role of PHA synthase, Nat. Prod. Rep. 20, 445457 .

8. Gerngross, T. U., Snell, K. D., Peoples, O. P., Sinskey, A. J., Cushai, E., Masamune, S., and Stubbe, J. (1994) Overexpression and purification of the soluble polyhydroxyalkanoate synthase 
from Alcaligenes eutrophus: Evidence for a required posttranslational modification for catalytic activity, Biochemistry 33, 93119320 .

9. Liebergesell, M., Sonomoto, K., Madkour, M., Mayer, F., and Steinbuchel, A. (1994) Purification and characterization of the poly(hydroxyalkanoic acid) synthase from Chromatium vinosum and localization of the enzyme at the surface of poly(hydroxyalkanoic acid) granules, Eur. J. Biochem. 226, 71-80.

10. Alonso, M. D., Lomako, J., Lomako, W. A., and Whelan, W. J. (1995) A new look at the biogenesis of glycogen, FASEB J. 9, 1126-1137.

11. Zeeman, S. C., Smith, S. M., and Smith, A. M. (2002) The priming of amylose synthesis in Arabidopsis leaves, Plant Physiol. 128, 1069-1076.

12. Ugalde, J. E., Parodi, A. J., and Ugalde, R. A. (2003) De novo synthesis of bacterial glycogen: Agrobacterium tumefaciens glycogen synthase is involved in glucan initiation and elongation, Proc. Natl. Acad. Sci. U.S.A. 100, 10659-10663.

13. Wodzinska, J., Snell, K. D., Rhomberg, A., Sinskey, A. J., Biemann, K., and Stubbe, J. (1996) Polyhydroxybutyrate synthase: Evidence for covalent catalysis, J. Am. Chem. Soc. 118, 6319-6320.

14. Müh, U., Sinskey, A. J., Kirby, D. P., Lane, W. S., and Stubbe, J. (1999) PHA synthase from Chromatium vinosum: Cysteine 149 is involved in covalent catalysis, Biochemistry 38, 826-837.

15. Tian, J., Sinskey, A. J., and Stubbe, J. (2005) Detection of Intermediates from the Polymerization Reaction Catalyzed by a D302A Mutant of Class III Polyhydroxyalkanoate (PHA) Synthase, Biochemistry 44, 1495-1503.

16. Yuan, W., Jia, Y., Tian, J., Snell, K. D., Muh, U., Sinskey, A. J., Lambalot, R. H., Walsh, C. T., and Stubbe, J. (2001) Class I and III polyhydroxyalkanoate synthases from Ralstonia eutropha and Allochromatium vinosum: characterization and substrate specificity studies, Arch. Biochem. Biophys. 394, 87-98.

17. Jia, Y., Kappock, T. J., Frick, T., Sinskey, A. J., and Stubbe, J. (2000) Lipases provide a new mechanistic model for polyhydroxybutyrate (PHB) synthases: Characterization of the functional residues in Chromatium vinosum PHB synthase, Biochemistry 39, 3927-3936.
18. Laemmli, U.K. (1970) Cleavage of structural proteins during the assembly of the head of bacteriophage T4, Nature 227, 680685.

19. Jossek, R., Reichelt, R., and Steinbüchel, A. (1998) In vitro biosynthesis of poly(hydroxybutyric acid) by using purified poly(hydroxyalkanoic acid) synthase of Chromatium vinosum, Appl. Microbiol. Biotechnol. 49, 258-266.

20. Jia, Y., Yuan, W., Wodzinska, J., Park, C., Sinskey, A. J., and Stubbe, J. (2001) Mechanistic studies of Class I polyhydroxybutyrate (PHB) synthase from Ralstonia eutropha: Class I and III synthases share a similar catalytic mechanism, Biochemistry 40, 1011-1019.

21. Bruner, S. D., Weber, T., Kohli, R. M., Schwarzer, D., Marahiel, M. A., Walsh, C. T., and Stubbs, M. T. (2002) Structural basis for the cyclization of the lipopeptide antibiotic surfactin by the thioesterase domain SrfTE, Structure 10, 301-310.

22. Rehm, B. H., Antonio, R. V., Spiekermann, P., Amara, A. A., and Steinbuchel, A. (2002) Molecular characterization of the poly(3-hydroxybutyrate) (PHB) synthase from Ralstonia eutropha: in vitro evolution, site-specific mutagenesis and development of a PHB synthase protein model, Biochim. Biophys. Acta 1594, 178190.

23. Tian, J., He, A., Lawrence, A., Liu, P., Watson, N., Sinskey, A. J., and Stubbe, J. (2005) Analysis of Transient Polyhydroxybutyrate Production in Wautersia eutropha H16 by Quantitative Westerns and Transmission Electron Microscopy, J. Bacteriol. (in press).

24. Kawaguchi, Y. and Doi, Y. (1992) Kinetics and Mechanism of Synthesis and Degradation of Poly(3-Hydroxybutyrate) in Alcaligenes eutrophus, Macromolecules 25, 2324-2329.

25. Madden, L. A., Anderson, A. J., Shah, D. T., and Asrar, J. (1999) Chain termination in polyhydroxyalkanoate synthesis: Involvement of exogenous hydroxy-compounds as chain transfer agents, Int. J. Biol. Macromol. 25, 43-53.

26. Ahn, K. and Kornberg, A. (1990) Polyphosphate kinase from Escherichia coli. Purification and demonstration of a phosphoenzyme intermediate, J. Biol. Chem. 265, 11734-11739.

BI050331U 